\title{
RANDOM TREES WITH SUPEREXPONENTIAL BRANCHING WEIGHTS
}

\author{
SVANTE JANSON, THORDUR JONSSON, AND SIGURDUR ÖRN STEFÁNSSON
}

\begin{abstract}
We study rooted planar random trees with a probability distribution which is proportional to a product of weight factors $w_{n}$ associated to the vertices of the tree and depending only on their individual degrees $n$. We focus on the case when $w_{n}$ grows faster than exponentially with $n$. In this case the measures on trees of finite size $N$ converge weakly as $N$ tends to infinity to a measure which is concentrated on a single tree with one vertex of infinite degree. For explicit weight factors of the form $w_{n}=((n-1) !)^{\alpha}$ with $\alpha>0$ we obtain more refined results about the approach to the infinite volume limit.
\end{abstract}

\section{INTRODUCTION}

Random trees have been studied intensively by mathematicians and theoretical physicists in the last few decades. They have direct applications to many branches in science, they are essential in many mathematical models used by physicists and are a natural object to study from the point of view of pure mathematics.

The random trees we are concerned with here were originally called simply generated trees by probabilists [9]. Later the same tree ensembles were referred to as random trees with a local action by physicists and viewed as toy models in statistical mechanics and for some aspects of quantum gravity, see e.g. [1].

Simply generated trees with $N$ vertices can be defined as follows: Let $\left(w_{n}\right)_{n \geq 1}$ be a sequence of nonnegative numbers which we will call branching weights. If $T$ is a tree graph with vertex set $V(T)$ having $N$ elements we define a probability distribution on the set of all such trees by the formula

$$
\nu(T)=Z^{-1} \prod_{v \in V(T)} w_{\sigma(v)}
$$

where $\sigma(v)$ is the degree of the vertex $v$ and $Z$ is a normalization factor called partition function in physics. One is interested in typical properties of trees with respect to this measure, especially asymptotics for large $N$ and the existence of a limiting measure as $N \rightarrow \infty$.

Date: 14 May, 2011.

2000 Mathematics Subject Classification. 05C80, 05C05, 60J80, 60F05.

Key words and phrases. Random trees, simply generated trees, branching process, weak limit. 
A lot is known about such trees for "nice" branching weights as we review briefly below. In this paper we aim at complementing some of these results for weights $w_{n}$ which grow faster than exponentially with $n$. In this case some of the formalism that has been used to study simply generated trees is not applicable any more as we will explain below. A physicist would say that the Grand partition function is divergent which normally is a signal of instability in a physical theory. We will indeed see that with superexponential branching weights one vertex becomes connected to all the other vertices in the infinite volume limit.

In the next section we give a more technical background and summarize our results. The final section contains detailed proofs.

\section{Definitions And SUmmary of Results}

We consider rooted planar trees with root $r$ of degree 1 . We let $\Gamma_{N}$ be the set of trees with $N$ edges and denote the set of finite and infinite trees by $\Gamma$. Vertices of infinite order are allowed and for such vertices the links pointing away from the root are ordered as $\mathbb{N}$, i.e. there is a leftmost edge pointing away from the root. The unique nearest neighbour of the root $r$ will be denoted by $s$.

Remark 2.1. We include the root $r$ just for convenience. It is equivalent to omit it and consider $s$ as the root (now with arbitrary degree), with minor changes in the notation; $N$ is then the number of vertices in the tree and the degree $\sigma(v)$ is replaced by $1+\sigma_{+}(v)$ where $\sigma_{+}(v)$ is the outdegree of $v$. It may be even more convenient to omit $r$ but keep the pendant edge from $s$ to $r$ as an edge with one free endpoint; this point of view is used sometimes in the proofs below.

Remark 2.2. We can regard the set $\Gamma$ as a set of subtrees of the infinite Ulam-Harris tree $T_{\infty}$, which is the tree with vertex set $V\left(T_{\infty}\right)=\{r\} \cup$ $\bigcup_{k=0}^{\infty} \mathbb{N}^{k}$, the set of all finite strings of natural numbers (and $r$ ), with $s=\emptyset$ (the empty string, so $\mathbb{N}^{0}=\{s\}$ ) and a vertex $v=v_{1} \cdots v_{k}$ having ancestor $v_{1} \cdots v_{k-1}$ when $k>0$. More precisely, $\Gamma$ can be identified with the set of all rooted subtrees $T$ of $T_{\infty}$ such that if $v=v_{1} \cdots v_{k}$ is a vertex in $T$, then so is $v_{1} \cdots v_{k-1} i$ for every $i<v_{k}$. We call such subtrees of $T_{\infty}$ left subtrees and more generally, we say that a tree $T^{\prime} \in \Gamma$ is a left subtree of $T \in \Gamma$ if $V\left(T^{\prime}\right) \subseteq V(T)$.

We endow $\Gamma$ with a metric $d$ which is defined as follows: Let $T \in \Gamma$ and define $B_{R}(T)$ as the graph ball of radius $R$, centered on the root $r$ in $T$. The left ball of radius $R, L_{R}(T)$, is defined as the maximal left subtree of $B_{R}(T)$ with vertices of degree no greater than $R$. The metric $d$ is given by

$$
d\left(T, T^{\prime}\right)=\inf \left\{\frac{1}{R+1} \mid L_{R}(T)=L_{R}\left(T^{\prime}\right)\right\}, \quad T, T^{\prime} \in \Gamma .
$$


Convergence in $\Gamma$, in the metric $d$, is equivalent to convergence of the degree $\sigma(v)$ for every $v \in V\left(T_{\infty}\right)$ (where we define $\sigma(v)=0$ for $v \notin T$ ), see [6] for details.

To avoid trivialities we assume that the branching weights satisfy $w_{1} \neq 0$ and $w_{n} \neq 0$ for at least some $n>2$. We define the finite volume partition function

$$
Z_{N}=\sum_{\tau \in \Gamma_{N}} \prod_{v \in V(\tau) \backslash\{r\}} w_{\sigma(v)}
$$

and a probability distribution $\nu_{N}$ on $\Gamma_{N}$ by

$$
\nu_{N}(\tau)=Z_{N}^{-1} \prod_{v \in V(\tau) \backslash\{r\}} w_{\sigma(v)} .
$$

This probability distribution describes a random tree $T_{N}$ with $N$ edges.

Let $\rho \geq 0$ be the radius of convergence of the generating function

$$
g(z)=\sum_{n=0}^{\infty} w_{n+1} z^{n}
$$

of the branching weights. A rescaling $w_{n} \mapsto a b^{n} w_{n}$ with $a, b>0$ does not affect the distributions $\nu_{N}$, and it is well-known and easy to see that if $\rho>0$, we can by rescaling assume that $\left(w_{n}\right)$ is a probability distribution, i.e. $\sum_{0}^{\infty} w_{n}=1$. In that case, the random tree $T_{N}$ with distribution $\nu_{N}$ is a Galton-Watson tree with offspring distribution $\left(w_{n+1}\right)_{n=0}^{\infty}$, conditioned to have size $N$. If further $\lim _{z} \jmath_{\rho} z g^{\prime}(z) / g(z) \geq 1$, then the distributions $\nu_{N}$ converge to the distribution of a random tree that is infinite, with all vertex degrees finite and exactly one infinite path, see further [2, 3, 8]. The limiting measure describes an infinite critical Galton-Watson tree conditioned on nonextinction. On the other hand, in the subcritical case when $m=\lim _{z \nearrow \rho} z g^{\prime}(z) / g(z)<1$, then (at least under some technical conditions) the limit distribution still exists but now describes a random tree with exactly one vertex of infinite degree; the length of the path from $r$ to this vertex has a geometric distribution with mean $1 /(1-m)$; the rest of the tree can be described by a subcritical Galton-Watson process, see [6] for details.

In the present paper we are interested in the case when the radius of convergence $\rho=0$. Note that then there is no Galton-Watson interpretation. We prove in Section 3 weak convergence, as $N \rightarrow \infty$, of the measures $\nu_{N}$ (in the topology generated by $d$ ) in this case too, under certain conditions on the weights. The result can be seen as a natural limiting case of the result in [6] as $m \rightarrow 0$; the resulting limit tree is in this case non-random, and is simply an infinite star.

Theorem 2.3. If the branching weights satisfy

$$
\frac{w_{n+1}}{w_{n}} \underset{n \rightarrow \infty}{\longrightarrow} \infty
$$


then the measures $\nu_{N}$ viewed as probability measures on $\Gamma$, converge weakly to the probability measure that is concentrated on the single tree which has $\sigma(s)=\infty$ and all other vertices of degree one.

Furthermore, we obtain stronger convergence results for certain explicit choices of weights. In the language of statistical mechanics these results give an explicit description of the finite size effects.

Theorem 2.4. For the branching weights $w_{2}=\lambda$ and $w_{n}=(n-1) !, n \neq 2$, the partition function satisfies

$$
\frac{Z_{N}}{e^{\lambda}(N-1) !} \rightarrow 1
$$

and

$$
N-\sigma(s) \stackrel{d}{\longrightarrow} \operatorname{Pois}(\lambda)
$$

as $N \rightarrow \infty$. Moreover, the tree $T_{N}$ consists of $r, s$, and $\sigma(s)-1$ branches attached to $s$; with probability tending to $1, N-\sigma(s)$ of these branches have size 2 and all other have size 1 (i.e., they contain a single leaf only).

Note that in the limit $N \rightarrow \infty$, the branches of size 2 disappear to infinity, so we do not see them in the limit given by Theorem 2.3 .

Theorem 2.5. Let the branching weights be $w_{n}=((n-1) !)^{\alpha}$, where $0<$ $\alpha<1$. Then the partition function satisfies

$$
Z_{N}=((N-1) !)^{\alpha} \exp \left(O\left(N^{1-\alpha}\right)\right)=\exp \left(\alpha N \log (N)-\alpha N+O\left(N^{1-\alpha}\right)\right) .
$$

Furthermore, with probability tending to 1 , the random tree $T_{N}$ has the following properties, with $K=\lfloor 1 / \alpha\rfloor$ :

(i) $\sigma(s)=N-O\left(N^{1-\alpha}\right)$.

(ii) All vertices except $s$ have degrees $\leq K+1$.

(iii) All subtrees attached to $s$ have sizes $\leq K+1$.

Moreover, let $X_{i, N}$ be the number of vertices of degree $i$ in $T_{N}$ and let

$$
n_{i}=i !^{\alpha} N^{1-i \alpha} \text {. }
$$

(iv) If $1 \leq i<1 / \alpha$, then $n_{i} \rightarrow \infty$ as $N \rightarrow \infty$ and

$$
\frac{X_{i+1, N}}{n_{i}} \stackrel{\mathrm{p}}{\longrightarrow} 1 \text {. }
$$

If $i=1 / \alpha=K$ (which occurs only when $1 / \alpha$ is an integer), then $n_{K}=K !^{\alpha}$ is constant and

$$
X_{K+1, N} \stackrel{\mathrm{d}}{\longrightarrow} \operatorname{Pois}\left(n_{K}\right) .
$$

With these branching weights, the asymptotic distributions of the numbers $X_{i, N}$ of vertices of different degrees are Gaussian, except in the Poisson case when (2.11) applies. 
Theorem 2.6. Let $w_{n}=((n-1) !)^{\alpha}$ with $0<\alpha<1$ as in Theorem 2.5. Then there exist numbers $n_{i}^{*}=n_{i}^{*}(N)=(1+o(1)) n_{i}, 1 \leq i<1 / \alpha$, with $n_{i}$ given by (2.9), such that, as $N \rightarrow \infty$,

$$
\begin{aligned}
\frac{X_{i+1, N}-n_{i}^{*}}{\sqrt{n_{i}}} \stackrel{\mathrm{d}}{\longrightarrow} \mathcal{N}(0,1), & 1 \leq i<1 / \alpha, \\
X_{i+1, N} \stackrel{\mathrm{d}}{\longrightarrow} \operatorname{Pois}\left(n_{i}\right), & i=K=1 / \alpha .
\end{aligned}
$$

Moreover, these hold jointly for all $i \leq K$, with independent limits.

More precisely, for each $i<1 / \alpha$,

$$
n_{i}^{*}=n_{i}\left(1-(1-i \alpha) N^{-\alpha}+O\left(N^{-2 \alpha}\right)\right)+O(1) .
$$

In particular, when $\alpha$ is not too small, we have the explicit limits

$$
\begin{aligned}
& \frac{X_{2, N}-N^{1-\alpha}}{N^{(1-\alpha) / 2}} \stackrel{\mathrm{d}}{\longrightarrow} \mathcal{N}(0,1), \quad 1>\alpha>\frac{1}{3}, \\
& \frac{X_{2, N}-\left(N^{1-\alpha}-(1-\alpha) N^{1-2 \alpha}\right)}{N^{(1-\alpha) / 2}} \stackrel{\mathrm{d}}{\longrightarrow} \mathcal{N}(0,1), \quad 1>\alpha>\frac{1}{5} \text {, } \\
& \frac{X_{3, N}-2^{\alpha} N^{1-2 \alpha}}{N^{(1-2 \alpha) / 2}} \stackrel{\mathrm{d}}{\longrightarrow} \mathcal{N}\left(0,2^{\alpha}\right), \quad \frac{1}{2}>\alpha>\frac{1}{4}, \\
& \frac{X_{3, N}-\left(2^{\alpha} N^{1-2 \alpha}-(1-2 \alpha) 2^{\alpha} N^{1-3 \alpha}\right)}{N^{(1-2 \alpha) / 2}} \stackrel{\mathrm{d}}{\longrightarrow} \mathcal{N}\left(0,2^{\alpha}\right), \quad \frac{1}{2}>\alpha>\frac{1}{6} \text {, } \\
& \frac{X_{4, N}-6^{\alpha} N^{1-3 \alpha}}{N^{(1-3 \alpha) / 2}} \stackrel{\mathrm{d}}{\longrightarrow} \mathcal{N}\left(0,6^{\alpha}\right), \quad \frac{1}{3}>\alpha>\frac{1}{5}, \\
& \frac{X_{4, N}-\left(6^{\alpha} N^{1-3 \alpha}-(1-3 \alpha) 6^{\alpha} N^{1-4 \alpha}\right)}{N^{(1-3 \alpha) / 2}} \stackrel{\mathrm{d}}{\longrightarrow} \mathcal{N}\left(0,6^{\alpha}\right), \quad \frac{1}{3}>\alpha>\frac{1}{7} .
\end{aligned}
$$

For smaller $\alpha$, it is possible to obtain further terms in the expansion of $n_{i}^{*}$, and thus explicit forms of the asymptotic mean of $X_{i+1, N}$. However, this approach seems to become more and more difficult as $\alpha$ becomes smaller.

Remark 2.7. The proof of (2.12) shows also the stronger result that the joint distribution of $\left(X_{i+1, N}\right)_{i=1}^{K}$ can be approximated by the joint distribution of independent Poisson random variables $Y_{i, N} \sim \operatorname{Pois}\left(n_{i}^{*}\right)$, in the sense that the total variation distance tends to 0 as $N \rightarrow \infty$ :

$$
\frac{1}{2} \sum_{m_{1}, \ldots, m_{K}}\left|\mathbb{P}\left(X_{i+1, N}=m_{i}, \forall i\right)-\mathbb{P}\left(Y_{i, N}=m_{i}, \forall i\right)\right| \rightarrow 0 .
$$

Remark 2.8. The estimate (2.8) of the partition function can be improved to

$$
Z_{N}=(N-1) !^{\alpha} \exp \left(N^{1-\alpha}+\left(2^{\alpha}-\frac{1-\alpha}{2}\right) N^{1-2 \alpha}+O\left(N^{1-3 \alpha}\right)+o(1)\right) .
$$

In particular, if $1>\alpha>\frac{1}{2}$, then $Z_{N}=(N-1) !^{\alpha} \exp \left(N^{1-\alpha}+o(1)\right)$.

Again it seems possible, but more complicated, to obtain further terms in the exponent. 
Remark 2.9. It is straightforward to show, using the same methods as in the proof of Theorem 2.4, that when $\alpha>1$

$$
Z_{N}=(N-1) !^{\alpha}(1+o(1))
$$

and all the branches which are attached to $s$ have size 1, with a probability which tends to 1 as $N \rightarrow \infty$. In this case the leading contribution to the partition function comes only from the Boltzmann factor of the vertex $s$, i.e. $w_{\sigma(s)}$. The case $\alpha=1$ is a marginal case when larger branches start to appear and their entropy adds a contribution to the partition function which appears in the associated exponential.

\section{Proofs of theorems}

In this section we state and prove a few lemmas and prove Theorems 2.32 .5 . In the following we will always assume that the branching weights satisfy the condition in Equation (2.5). Define

$$
Z(N, n)=\sum_{d_{1}+\cdots+d_{N}=n} \prod_{i=1}^{N} w_{d_{i}+1}
$$

By Lagrange's inversion formula [6, 5] (or by a combinatorial argument, see [4, 7, 10]), it holds that

$$
Z_{N}=\frac{1}{N} Z(N, N-1)
$$

More generally the partition function for an ordered forest of $m$ trees with a total number of edges $N$ is

$$
Z_{N}^{(m)}=\frac{m}{N} Z(N, N-m) .
$$

Lemma 3.1. For every $\epsilon>0$ there exists a $C_{\epsilon}<\infty$ such that for all $N$ and $n$

$$
Z(N, n) \leq \epsilon Z(N, n+1)+C_{\epsilon}^{N}
$$

Proof. Consider a finite sequence $d_{1}, \ldots, d_{N}$ for which $\sum_{i} d_{i}=n$. Let $i^{*}$ be the smallest index such that $d_{i^{*}}=\max _{i} d_{i}$. Define a sequence

$$
d_{i}^{*}= \begin{cases}d_{i}+1 & \text { if } i=i^{*} \\ d_{i} & \text { otherwise } .\end{cases}
$$

Note that $d_{i^{*}}^{*}$ is the unique maximum in $\left(d_{i}^{*}\right)$, so $\left(d_{i}\right)$ can be recovered from $\left(d_{i}^{*}\right)$ and the map $\left(d_{i}\right) \mapsto\left(d_{i}^{*}\right)$ is injective.

Let $\epsilon>0$ be given. Choose a number $A_{\epsilon}$ such that $w_{i} / w_{i+1}<\epsilon$ if $i \geq A_{\epsilon}$. Then

$$
\sum_{\substack{d_{1}+\cdots+d_{N}=n \\ \max _{i} d_{i}>A_{\epsilon}}} \prod_{i=1}^{N} w_{d_{i}+1} \leq \epsilon \sum_{d_{1}^{*}+\cdots+d_{N}^{*}=n+1} \prod_{i=1}^{N} w_{d_{i}^{*}+1} \leq \epsilon Z(N, n+1)
$$


and, crudely,

$$
\sum_{\substack{d_{1}+\cdots+d_{N}=n \\ \max _{i} d_{i} \leq A_{\epsilon}}} \prod_{i=1}^{N} w_{d_{i}+1} \leq\left(\sum_{i=0}^{A_{\epsilon}} w_{i+1}\right)^{N} .
$$

Taking $C_{\epsilon}=\sum_{i=0}^{A_{\epsilon}} w_{i+1}$ completes the proof.

Lemma 3.2. As $N \rightarrow \infty, \sigma(s) \stackrel{p}{\rightarrow} \infty$.

Proof. It suffices to show that

$$
\nu_{N}(\sigma(s)=k) \rightarrow 0
$$

for every fixed $k \geq 1$, since $\nu_{N}(\sigma(s) \geq m)=1-\sum_{k=1}^{m-1} \nu_{N}(\sigma(s)=k)$. If the vertex $s$, in a tree with $N$ edges, has degree $k+1$, then removing $s$ and $r$ but leaving all edges from $s$ to its children as pendant edges, cf. Remark 2.1, leaves a forest with $k$ trees and $N-1$ edges. Therefore, using (3.2) and (3.3),

$$
\nu_{N}(\sigma(s)=k+1)=\frac{N}{N-1} k w_{k+1} \frac{Z(N-1, N-k-1)}{Z(N, N-1)} .
$$

Let $\epsilon>0$ be given. Use Lemma $3.1 k$ times to get

$$
Z(N-1, N-k-1) \leq \epsilon^{k} Z(N-1, N-1)+k C_{\epsilon}^{N}
$$

and note that

$$
Z(N, N-1) \geq w_{1} Z(N-1, N-1) .
$$

Since the branching weights satisfy (2.5),$Z(N-1, N-1) \geq w_{N} w_{1}^{N-2}$ grows super exponentially and in particular $Z(N-1, N-1) \geq\left(2 C_{\epsilon}\right)^{N}$ for $N$ large enough. Therefore

$$
\nu_{N}(\sigma(s)=k+1) \leq \frac{N}{N-1} k w_{k+1}\left(w_{1}^{-1} \epsilon^{k}+k 2^{-N}\right)
$$

and (3.8) follows since $\epsilon$ is arbitrary.

Lemma 3.3. For any $N \geq 1$ and $n \geq 0$

$$
\sum_{\ell=0}^{N} \ell w_{\ell+1} Z(N-1, n-\ell)=\frac{n}{N} Z(N, n) .
$$

Proof.

$$
\begin{aligned}
& \sum_{\ell=0}^{n} \ell w_{\ell+1} Z(N-1, n-\ell)=\sum_{d_{1}+\cdots+d_{N-1}+\ell=n} \ell w_{\ell+1} \prod_{i=1}^{N-1} w_{d_{i}+1} \\
& =\sum_{d_{1}+\cdots+d_{N}=n} d_{N} \prod_{i=1}^{N} w_{d_{i}+1} .
\end{aligned}
$$

By symmetry we can replace $d_{N}$ in front of the product by any $d_{j}, j=$ $1, \ldots, N$. Summing over $j$ then gives the desired result. 
Lemma 3.4. Assume $N>1$ and let $s_{1}$ be the first child of $s$. If $L \geq 1$ and $k \geq L$, then

$$
\nu_{N}\left(L+1 \leq \sigma\left(s_{1}\right) \leq k+1 \mid \sigma(s)=k+1\right) \leq \frac{2}{L} .
$$

Proof. If $\sigma(s)=k+1 \geq 2$ and $\sigma\left(s_{1}\right)=\ell+1 \geq 1$ then removing the vertices $r, s$ and $s_{1}$, again leaving pendant edges, leaves a forest with $k+\ell-1$ trees and $N-2$ edges. Therefore (assuming $N \geq 3$ ),

$$
\begin{aligned}
\nu_{N}\left(\sigma(s)=k+1, \sigma\left(s_{1}\right)=\ell+1\right) \\
\\
=\frac{N(k+\ell-1) w_{k+1} w_{\ell+1}}{N-2} \frac{Z(N-2, N-1-k-\ell)}{Z(N, N-1)} .
\end{aligned}
$$

By (3.9) and (3.16),

$$
\begin{aligned}
\nu_{N}\left(\sigma\left(s_{1}\right)=\ell\right. & +1 \mid \sigma(s)=k+1) \\
& =\frac{(N-1)(k+\ell-1) w_{\ell+1}}{(N-2) k} \frac{Z(N-2, N-1-k-\ell)}{Z(N-1, N-1-k)} .
\end{aligned}
$$

By Lemma 3.3 ,

$$
\begin{aligned}
\sum_{\ell \geq L} w_{\ell+1} Z(N-2, N-1-k-\ell) & \leq \frac{1}{L} \sum_{\ell \geq 0} \ell w_{\ell+1} Z(N-2, N-1-k-\ell) \\
& =\frac{1}{L} \frac{N-1-k}{N-1} Z(N-1, N-1-k) .
\end{aligned}
$$

Hence, (3.17) implies

$$
\sum_{\ell=L}^{k} \nu_{N}\left(\sigma\left(s_{1}\right)=\ell+1 \mid \sigma(s)=k+1\right) \leq \frac{N-1-k}{N-2} \frac{2}{L} \leq \frac{2}{L} .
$$

Lemma 3.5. As $N \rightarrow \infty, \nu_{N}\left(\sigma\left(s_{1}\right)=1\right) \rightarrow 1$.

Proof. Fix $L>1$ and an $\ell$ such that $1 \leq \ell<L$. Note that when $\ell \geq 1$ the formula (3.16) is symmetric in $k$ and $\ell$. Therefore

$$
\begin{aligned}
\nu_{N}\left(\sigma\left(s_{1}\right)=\ell+1\right) & =\sum_{k=1}^{\infty} \nu_{N}\left(\sigma(s)=k+1, \sigma\left(s_{1}\right)=\ell+1\right) \\
& =\nu_{N}\left(\sigma(s)=\ell+1, \sigma\left(s_{1}\right) \geq 2\right) \leq \nu_{N}(\sigma(s)=\ell+1)
\end{aligned}
$$


and thus $\nu_{N}\left(\sigma\left(s_{1}\right)=\ell+1\right) \rightarrow 0$ as $N \rightarrow \infty$ by Lemma 3.2. Next, Lemma 3.4 implies

$$
\begin{aligned}
& \nu_{N}\left(L+1 \leq \sigma\left(s_{1}\right) \leq \sigma(s)\right) \\
& \quad=\sum_{k} \nu_{N}\left(L+1 \leq \sigma\left(s_{1}\right) \leq k+1 \mid \sigma(s)=k+1\right) \nu_{N}(\sigma(s)=k+1) \leq \frac{2}{L} .
\end{aligned}
$$

Thus

$$
\begin{aligned}
\limsup _{N \rightarrow \infty} \nu_{N}\left(2 \leq \sigma\left(s_{1}\right) \leq \sigma(s)\right) & \leq \limsup _{N \rightarrow \infty}\left(\sum_{\ell=1}^{L-1} \nu_{N}\left(\sigma\left(s_{1}\right)=\ell+1\right)+\frac{2}{L}\right) \\
& =\frac{2}{L} .
\end{aligned}
$$

Since $L$ is arbitrary, $\nu_{N}\left(2 \leq \sigma\left(s_{1}\right) \leq \sigma(s)\right) \rightarrow 0$ as $N \rightarrow \infty$. By the symmetry of (3.16) in $k$ and $\ell$ we also find that

$$
\nu_{N}\left(2 \leq \sigma(s) \leq \sigma\left(s_{1}\right)\right)=\nu_{N}\left(2 \leq \sigma\left(s_{1}\right) \leq \sigma(s)\right) \rightarrow 0
$$

as $N \rightarrow \infty$. Finally, since $\sigma(s) \geq 2$, we have

$$
\nu_{N}\left(\sigma\left(s_{1}\right) \geq 2\right) \leq \nu_{N}\left(2 \leq \sigma(s) \leq \sigma\left(s_{1}\right)\right)+\nu_{N}\left(2 \leq \sigma\left(s_{1}\right) \leq \sigma(s)\right) \rightarrow 0
$$

as $N \rightarrow \infty$.

Proof of Theorem [2.3. Let $R>0$. By Lemma 3.2, $\sigma(s) \stackrel{\mathrm{p}}{\longrightarrow} \infty$, so $\nu_{N}(\sigma(s) \geq$ $R) \rightarrow 1$. Given that $\sigma(s) \geq R$, denote the first $R-1$ children of $s$ by $s_{1}, \ldots, s_{R-1}$. Then by Lemma 3.5 and symmetry $\nu_{N}\left(\sigma\left(s_{i}\right)=1, \sigma(s) \geq\right.$ $R) \rightarrow 1$ for every $i \leq R$ and thus we find that

$$
\nu_{N}\left(\sigma(s) \geq R, \sigma\left(s_{1}\right)=\cdots=\sigma\left(s_{R-1}\right)=1\right) \rightarrow 1
$$

as $N \rightarrow \infty$. Since $R$ is arbitrary, the result follows from the definition of the topology on $R$, cf. the comment below (2.1).

Proof of Theorem 2.4. First, we establish an upper bound on $Z_{N}$. Consider Equation (3.2) for $Z_{N}$. For a given sequence $\left(d_{i}\right)$, let $m_{j}$ denote the number of indices $i$ for which $d_{i}=j$ where $j=0, \ldots, N-1$. Instead of summing over $\left(d_{i}\right)$ we sum over $\left(m_{j}\right)$. For a given sequence $\left(m_{j}\right)$ there are $\left(\begin{array}{c}N \\ m_{0}, \ldots, m_{N-1}\end{array}\right)$ sequences $\left(d_{i}\right)$ and therefore, since $w_{1}=1$,

$$
\begin{aligned}
\frac{Z_{N}}{(N-1) !} & =\sum_{\substack{m_{0}+\cdots+m_{N-1}=N \\
m_{1}+2 m_{2}+\cdots+(N-1) m_{N-1}=N-1}} \prod_{i=0}^{N-1} \frac{w_{i+1}^{m_{i}}}{m_{i} !} \\
& =\sum_{\substack{m_{1}+2 m_{2}+\cdots+(N-1) m_{N-1}=N-1 \\
\left(N-\sum_{j=1}^{N-1} m_{j}\right) !}} \frac{1=1}{\prod_{i=1}^{N-1}} \frac{w_{i+1}^{m_{i}}}{m_{i} !} .
\end{aligned}
$$


Denote the maximum vertex degree by $M$ and fix a number $K \geq 2$. By Lemma 3.2, it is sufficient to consider the case $M>K$. That contribution to (3.26) can be estimated by shifting $m_{M-1} \rightarrow m_{M-1}+1$ which yields the upper bound

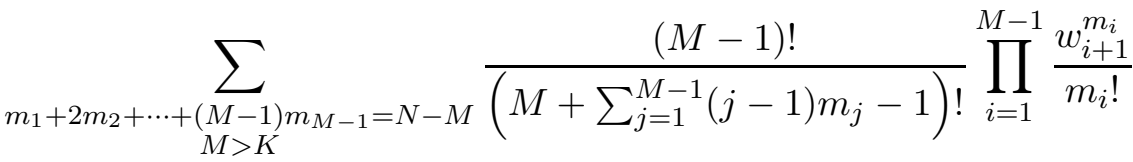

$$
\begin{aligned}
& \leq \sum_{m_{1}+2 m_{2}+\cdots+\left(\begin{array}{c}
M-1) m_{M-1}=N-M \\
M>K
\end{array}\right.} \frac{\lambda^{m_{1}}}{m_{1} !} \prod_{i=2}^{M-1} \frac{\left(i ! / M^{i-1}\right)^{m_{i}}}{m_{i} !} \\
& \leq \exp \left(\lambda+\sum_{i=2}^{\infty} \frac{i !}{(i \vee K)^{i-1}}\right)
\end{aligned}
$$

where $A \vee B$ denotes the maximum of $A$ and $B$. The last expression converges to $e^{\lambda}$ when $K \rightarrow \infty$ by dominated convergence.

Next we establish a corresponding lower bound on $Z_{N}$. Consider the contribution to (3.26) from terms for which the only nonzero elements in the sequence $\left(m_{i}\right)$ are $m_{0}, m_{1}$ and $m_{k}=1$ where $k \geq 2$ is arbitrary; thus $m_{0}=k, m_{1}=N-k-1$ and $m_{k}=1$. These terms provide the following lower bound of (3.26)

$$
\sum_{k=2}^{N-1} w_{k+1} \frac{1}{k !} \frac{w_{2}^{N-1-k}}{(N-1-k) !}=\sum_{\ell=0}^{N-3} \frac{\lambda^{\ell}}{\ell !} \rightarrow e^{\lambda}
$$

as $N \rightarrow \infty$. This and (3.27) prove (2.6).

To complete the proof, note that the probability that $T_{N}$ has $\sigma(s)=N-j$ and that exactly $j$ of the $\sigma(s)-1=N-j-1$ branches attached to $s$ have size 2 and all others size 1 is, assuming $N>2 j$ and using (2.6),

$\frac{1}{Z_{N}}\left(\begin{array}{c}N-j-1 \\ j\end{array}\right) w_{1}^{N-j-1} w_{2}^{j} w_{N-j}=\frac{1}{Z_{N}}\left(\begin{array}{c}N-j-1 \\ j\end{array}\right) \lambda^{j}(N-j-1) ! \rightarrow \frac{\lambda^{j}}{j !} e^{-\lambda}$.

These limits sum to 1 and yield the $\operatorname{Pois}(\lambda)$ distribution in (2.7).

Proof of Theorem 2.5. Consider the weights $w_{n+1}=n !^{\alpha}$. Write, again by (3.2),

$$
Z_{N}=\frac{1}{N} \sum_{d_{1}+\cdots+d_{N}=N-1} \prod_{i=1}^{N} d_{i} !^{\alpha} .
$$

We get the lower bound

$$
Z_{N} \geq(N-1) !^{\alpha}
$$

by considering only the terms in $Z_{N}$ with one $d_{i}=N-1$, and all others 0 (i.e., stars).

Define $Z_{N}(k, \epsilon)$ as the contribution to $Z_{N}$ when precisely $k \geq 0$ vertices have degree greater than $\epsilon(N-1)$ where $\epsilon$ is some small positive number. 
First consider the case when $k=0$. Let $\left(d_{i}\right)_{i=1}^{N}$ be a sequence which satisfies $d_{i} \leq \epsilon(N-1)$ for all $i$. Using the simple relation

$$
N ! M ! \leq(N+1) !(M-1) !, \quad \text { for } N \geq M-1
$$

we can distribute and add the smallest elements in $\left(d_{i}\right)_{i=1}^{N}$ to the larger ones until each of them reaches $\epsilon(N-1)$. Thus we obtain the upper bound, using Stirling's formula,

$$
\prod_{i=1}^{N} d_{i} !^{\alpha} \leq\lceil\epsilon(N-1)\rceil !^{\alpha / \epsilon} \leq C_{1} N^{2 \alpha / \varepsilon}(N-1) !^{\alpha} \epsilon^{\alpha N}
$$

where $C_{1}>0$ is a number independent of $N$ (but, as other constants below, it may depend on $\alpha$ and $\varepsilon$ ). Therefore,

$$
Z_{N}(0, \epsilon) \leq C_{1} N^{2 \alpha / \varepsilon}(N-1) !^{\alpha} \epsilon^{\alpha N}\left(\begin{array}{c}
2 N-2 \\
N-1
\end{array}\right) \leq C_{1} N^{2 \alpha / \varepsilon}(N-1) !^{\alpha} \epsilon^{\alpha N} 2^{2 N}
$$

which is negligible compared to (3.31) as $N \rightarrow \infty$ for $\epsilon$ small enough.

Next consider the case when two or more of the $d_{i}$ are larger than $\epsilon(N-1)$, i.e. when $k \geq 2$ in $Z_{N}(k, \epsilon)$. Clearly, $k<1 / \epsilon$. Denote the $d_{i}$ which are greater than $\epsilon(N-1)$ by $d_{i_{1}}, \ldots, d_{i_{k}}$ and let $D_{j}=d_{i_{j}}$. The indices $i_{j}$ can be chosen in $\left(\begin{array}{l}N \\ k\end{array}\right)$ ways. We will now lump together all the $D_{i}$ into a single one, i.e. we define

$$
D=D_{1}+\cdots+D_{k}
$$

For each $D$, there are at most $\left(\begin{array}{c}D+k-1 \\ k-1\end{array}\right)$ choices of $D_{1}, \ldots, D_{k}$. Note that, with $D_{*}=\lceil\varepsilon(N-1)\rceil$, using $D_{i} \geq D_{*}$ and Stirling's formula again,

$$
\frac{D_{1} ! \cdots D_{k} !}{D !} \leq \frac{D_{*}^{k}}{\left(k D_{*}\right) !} \leq C_{2} N^{k}\left(\frac{1}{k}\right)^{k \epsilon N}
$$

where $C_{2}>0$ is independent of $N$. Thus, we get the upper bound

$$
\begin{aligned}
& \sum_{2 \leq k \leq 1 / \epsilon} Z_{N}(k, \epsilon) \leq C_{2}^{\alpha} \sum_{2 \leq k \leq 1 / \epsilon}\left(\begin{array}{c}
N \\
k
\end{array}\right) N^{\alpha k}\left(\frac{1}{k}\right)^{\alpha k \epsilon N} \\
& \times \sum_{\substack{D+d_{1}+\cdots+d_{N-k}=N-1 \\
D>\epsilon(N-1), d_{i} \leq \epsilon(N-1), \forall i}}\left(\begin{array}{c}
D+k-1 \\
k-1
\end{array}\right) D !^{\alpha} \prod_{i=1}^{N-k} d_{i} !^{\alpha} \\
& \leq C_{3} N^{3 / \epsilon}\left(\frac{1}{2}\right)^{2 \alpha \epsilon N} Z_{N}(1, \epsilon) .
\end{aligned}
$$

where $C_{3}>0$ is independent of $N$. This estimate, together with (3.34), shows that the main contribution to $Z_{N}$ for $N$ large comes from $Z_{N}(1, \epsilon)$. 
Finally, we consider $Z_{N}(1, \epsilon)$. Using the representation as in (3.26) we have, writing $L=\lfloor\varepsilon(N-1)\rfloor$ for convenience,

$$
\frac{Z_{N}(1, \epsilon)}{(N-1) !}=\sum_{D=L+1}^{N-1} \sum_{m_{1}+2 m_{2}+\cdots+L m_{L}=N-1-D} \frac{D !^{\alpha}}{\left(N-1-\sum_{j=1}^{L} m_{j}\right) !} \prod_{i=1}^{L} \frac{i !^{\alpha m_{i}}}{m_{i} !}
$$

where $D+1$ denotes the degree of the large vertex and $m_{i}$ denotes the number of vertices which have degree $i+1$. Consider one term in this sum and let $\widetilde{D}=D+\sum_{i=K+1}^{L} i m_{i}$, adding the outdegrees of all vertices which have degree greater than $K+1$ to the large vertex. Then

$$
\widetilde{D} ! \geq D ! \cdot D^{\sum_{i=K+1}^{L} i m_{i}} \geq D ! \cdot L^{\sum_{i=K+1}^{L} i m_{i}}
$$

and

$$
\left(N-1-\sum_{i=1}^{K} m_{i}\right) ! \leq\left(N-1-\sum_{i=1}^{L} m_{i}\right) ! \cdot N^{\sum_{i=K+1}^{L} m_{i}}
$$

Thus

$$
\frac{D !^{\alpha}}{\left(N-1-\sum_{i=1}^{L} m_{i}\right) !} \leq \frac{\widetilde{D} !^{\alpha} N^{\sum_{i=K+1}^{L} m_{i}}}{\left(N-1-\sum_{i=1}^{K} m_{i}\right) ! L^{\alpha \sum_{i=K+1}^{L} i m_{i}}}
$$

and

$$
\begin{aligned}
\frac{Z_{N}(1, \epsilon)}{(N-1) !} \leq & \sum_{\widetilde{D}=L+1}^{N-1} \sum_{m_{1}+\cdots+K m_{K}=N-1-\widetilde{D}} \sum_{m_{K+1}, \ldots, m_{L} \geq 0} \\
& \frac{\widetilde{D} !^{\alpha}}{\left(N-1-\sum_{j=1}^{K} m_{j}\right) !} \prod_{i=1}^{K} \frac{!^{\alpha m_{i}}}{m_{i} !} \prod_{i=K+1}^{L}\left(\frac{N i !^{\alpha}}{L^{i \alpha}}\right)^{m_{i}} \frac{1}{m_{i} !} .
\end{aligned}
$$

We have

$$
\begin{aligned}
\sum_{m_{K+1}, \ldots, m_{L}} \prod_{i=K+1}^{L}\left(\frac{N i !^{\alpha}}{L^{i \alpha}}\right)^{m_{i}} \frac{1}{m_{i} !} & =\prod_{i=K+1}^{L} \exp \left(\frac{N i !^{\alpha}}{L^{i \alpha}}\right) \\
& =\exp \left(\sum_{i=K+1}^{L} \frac{N i !^{\alpha}}{L^{i \alpha}}\right)
\end{aligned}
$$

Let, using $L=\lfloor\varepsilon(N-1)\rfloor>\varepsilon N / 2$ (assuming $N$ large),

$$
a_{i}=\frac{N i !^{\alpha}}{L^{i \alpha}} \leq \frac{2^{i \alpha} i !^{\alpha}}{\varepsilon^{i \alpha}} N^{1-i \alpha}
$$

Noting that $a_{i+1} / a_{i}=((i+1) / L)^{\alpha} \leq 1$ for $i<L$, we find

$$
\begin{aligned}
\sum_{i=K+1}^{L} a_{i} \leq(K+1) a_{K+1}+N a_{2 K+2} & =O\left(N^{1-(K+1) \alpha}\right)+O\left(N^{2-(2 K+2) \alpha}\right) \\
& =o(1)
\end{aligned}
$$


and thus from (3.41),

$$
\begin{aligned}
\frac{Z_{N}(1, \epsilon)}{(N-1) !} \leq(1+o(1)) \sum_{\widetilde{D}=L+1}^{N-1} & \sum_{m_{1}+\cdots+K m_{K}=N-1-\widetilde{D}} \\
& \frac{\widetilde{D} !^{\alpha}}{\left(N-1-\sum_{j=1}^{K} m_{j}\right) !} \prod_{i=1}^{K} \frac{i !^{\alpha m_{i}}}{m_{i} !} .
\end{aligned}
$$

The sum here is just the sum in (3.37) with $m_{i}=0$ for $i>K$, so we have shown that $Z_{N}(1, \varepsilon)$ is dominated by such terms. Recalling (3.34) and (3.36) we see that

$$
\frac{Z_{N}}{(N-1) !}=(1+o(1)) \sum_{m_{1}+\cdots+K m_{K}<N-L-1} \frac{\left(N-1-\sum_{j=1}^{K} j m_{j}\right) !^{\alpha}}{\left(N-1-\sum_{j=1}^{K} m_{j}\right) !} \prod_{i=1}^{K} \frac{i !^{\alpha m_{i}}}{m_{i} !}
$$

and that $Z_{N}$ is dominated by trees having exactly one vertex of degree $>\varepsilon(N-1)$ and all other vertices having degrees $\leq K+1$.

By Lemma 3.2, the contribution from trees with $\sigma(s) \leq K+1$ is negligible, so it suffices to consider the case when the unique vertex with high degree is $s$, which proves (ii).

To obtain the more precise results in (i) and (iv), fix $i \leq K$, fix $m_{j}$ for $j \neq i$, and denote the summand in (3.46) by $b\left(m_{i}\right)$. Increasing $m_{i}$ by 1 decreases $D=N-1-\sum_{j=1}^{K} j m_{j}$ by $i$ and, assuming still $D>L$ and recalling the definition of $n_{i}$ in (2.9),

$$
\frac{b\left(m_{i}+1\right)}{b\left(m_{i}\right)} \leq N L^{-i \alpha} \frac{i !^{\alpha}}{m_{i}+1} \leq C_{4} \frac{N^{1-i \alpha} i !^{\alpha}}{m_{i}+1}=C_{4} \frac{n_{i}}{m_{i}+1} .
$$

If $m_{i} \geq\left\lfloor 2 C_{4} n_{i}\right\rfloor$, this ratio is less than $1 / 2$. In particular,

$$
\sum_{m_{i} \geq 3 C_{4} n_{i}} b\left(m_{i}\right) \leq 2 b\left(\left\lfloor 3 C_{4} n_{i}\right\rfloor\right) \leq 2^{2-C_{4} n_{i}} b\left(\left\lfloor 2 C_{4} n_{i}\right\rfloor\right) .
$$

If $i<1 / \alpha$, then $n_{i} \rightarrow \infty$ as $N \rightarrow \infty$. Summing over all $m_{j}, j \neq i$, we see that the contribution to $Z_{N}$ from $m_{i} \geq 3 C_{4} n_{i}$ is negligible, so we may assume that $m_{i}<3 C_{4} n_{i}$. In the exceptional case $i=1 / \alpha$, we obtain by the same argument that we may assume $m_{i}<\log N$, say. In particular, since $n_{i}=O\left(N^{1-i \alpha}\right)=O\left(N^{1-\alpha}\right)$, we see that we may assume $\sigma(s)=D+1=$ $N-\sum_{j=1}^{K} j m_{j}=N-O\left(N^{1-\alpha}\right)$, which proves (i).

For the remaining terms, we now may use $D=N-o(N)$ to improve (3.47) to

$$
\frac{b\left(m_{i}+1\right)}{b\left(m_{i}\right)}=(1+o(1)) N \cdot N^{-i \alpha} \frac{i !^{\alpha}}{m_{i}+1}=(1+o(1)) \frac{n_{i}}{m_{i}+1} .
$$

Assume $i<1 / \alpha$ and let $\delta>0$. We can repeat the argument above, using (3.49) instead of (3.47) and $(1+\delta / 2) n_{i}$ instead of $2 C_{4} n_{i}$, and conclude that the terms with $m_{i} \geq(1+\delta) n_{i}$ are negligible. Similarly, (3.49) implies also 
that the terms with $m_{i} \leq(1-\delta) n_{i}$ are negligible. Hence, $Z_{N}$ is dominated by terms with $(1-\delta) n_{i}<m_{i}<(1+\delta) n_{i}$. Since $X_{i+1, N}=m_{i}$, this proves (iv) for $i<1 / \alpha$.

If $1 / \alpha$ is an integer and $i=K=1 / \alpha$, then it follows from (3.49) in the same way that $m_{K}$ is stochastically bounded and that $\nu_{N}\left\{m_{K}=m+\right.$ $1\} / \nu_{N}\left\{m_{K}=m\right\} \rightarrow n_{K} /(m+1)$ for every $m$, which implies that $m_{K} \stackrel{\mathrm{d}}{\longrightarrow}$ Pois $\left(n_{K}\right)$, completing the proof of (iv).

Furthermore, (3.47) implies, for all $m_{i}$ such that $D>L$,

$$
\frac{b\left(m_{i}\right)}{b(0)} \leq \frac{\left(C_{4} n_{i}\right)^{m_{i}}}{m_{i} !}
$$

Using this for each $i \leq K$, we see that the general summand in (3.46) is at $\operatorname{most} \prod_{i=1}^{K} \frac{\left(C_{4} n_{i}\right)^{m_{i}}}{m_{i} !}$, and thus (3.46) yields

$$
\frac{Z_{N}}{(N-1) !} \leq(1+o(1)) \frac{(N-1)^{\alpha}}{(N-1) !} \sum_{m_{1}, \ldots, m_{K}} \prod_{i=1}^{K} \frac{\left(C_{4} n_{i}\right)^{m_{i}}}{m_{i} !}
$$

and

$$
\frac{Z_{N}}{(N-1) !^{\alpha}} \leq(1+o(1)) \prod_{i=1}^{K} \exp \left(C_{4} n_{i}\right)=\exp \left(\sum_{i=1}^{K} C_{4} n_{i}+o(1)\right),
$$

which proves (2.8).

Finally, we show (iii), If $\tau$ is a tree in $\Gamma_{N}$ such that all vertices except $s$ have degrees $\leq K+1$, but some branch attached to $s$ has more than $K+1$ vertices, pick the first such branch and find, by breadth-first search, say, a subtree $\tau_{0}$ of that branch with exactly $K+2$ vertices. Rearrange the edges inside $\tau_{0}$ so that $\tau_{0}$ is replaced by a star with center adjacent to $s$; this produces a vertex of degree $K+2$. Let $\tau^{\prime} \in \Gamma_{N}$ be the result of making this change inside $\tau$. We have changed the degree of (at most) $K+2$ vertices, and since all old and new degrees are at most $2 K+1$, the weights of $\tau$ and $\tau^{\prime}$ differ by at most a constant factor. Furthermore, $\tau^{\prime}$ has exactly one vertex of degree $K+2$, and thus only a bounded number of trees $\tau$ can produce the same $\tau^{\prime}$. Consequently,

$$
\begin{aligned}
& \mathbb{P}\left(T_{n} \text { has a branch of size }>K+1\right) \\
& \leq \leq C_{5} \mathbb{P}\left(T_{n} \text { has a vertex } \neq s \text { of degree }>K+1\right),
\end{aligned}
$$

and this probability tends to 0 by (ii)

Proof of Theorem [2.6. Recall that $Z_{N}$ is given by (3.46), and that the significant terms have $m_{i}=(1+o(1)) n_{i}=O\left(N^{1-i \alpha}\right)$, except when $i=1 / \alpha$.

Let us first note that if $1 / \alpha$ is an integer and $i=K=1 / \alpha$, then, see the proof of Theorem 2.5. (3.49) implies that $X_{K+1, N}=m_{K}$ has an asymptotic Poisson distribution $\operatorname{Pois}\left(n_{K}\right)$, which further is asymptotically independent of $X_{i, N}, i \leq K$; furthermore, $\sum_{m_{K}} b\left(m_{K}\right)=\exp \left(n_{K}+o(1)\right) b(0)$. In the 
sequel we thus assume $m_{K}=0$ and sum only over $m_{i}, i<K$, when $i=$ $K=1 / \alpha$; we omit the trivial modifications below in this case.

Define, for a fixed $\eta \in(0,1), V=\prod_{i=1}^{n}\left[(1-\eta) n_{i},(1+\eta) n_{i}\right]$. In the sequel we consider only $\left(m_{i}\right)_{1}^{K} \in V$; recall that it suffices to sum over such $\left(m_{i}\right)$ in (3.46). For more compact notation, write

$$
A=\sum_{i=1}^{K} m_{i} \quad \text { and } \quad B=\sum_{i=1}^{K} i m_{i} .
$$

Note that $A$ and $B$ are $O\left(N^{1-\alpha}\right)$. Use Stirling's approximation on the first factor in the sum in (3.46) to get

$$
\begin{aligned}
& \frac{(N-1-B) !^{\alpha}}{(N-1-A) !} \\
& =\sqrt{\frac{(2 \pi(N-1-B))^{\alpha}}{2 \pi(N-1-A)}\left(\frac{N-1-B}{e}\right)^{\alpha(N-1-B)}\left(\frac{e}{N-1-A}\right)^{N-1-A}\left(1+O\left(N^{-1}\right)\right)} \\
& =\sqrt{(2 \pi(N-1))^{\alpha-1}}\left(\frac{N-1}{e}\right)^{(\alpha-1)(N-1)}(N-1)^{A-\alpha B} \\
& \quad \times \exp \left\{\alpha B-A+\alpha(N-1-B) \log \left(1-\frac{B}{N-1}\right)\right. \\
& \left.\quad-(N-1-A) \log \left(1-\frac{A}{N-1}\right)\right\}\left(1+O\left(N^{-\alpha}\right)\right) \\
& =(N-1) !^{\alpha-1}(N-1)^{A-\alpha B} \exp \left\{\sum_{j=2}^{K} \frac{\alpha B^{j}-A^{j}}{j(j-1)(N-1)^{j-1}}\right\}(1+o(1)) \\
& =(N-1) !^{\alpha-1} N^{A-\alpha B} \exp \left\{\sum_{j=2}^{K} \frac{\alpha B^{j}-A^{j}}{j(j-1) N^{j-1}}\right\}(1+o(1)),
\end{aligned}
$$

where in the last step we expanded the logarithms and kept only powers of $A$ and $B$ which contribute for large $N$, and then approximated $N-1$ by $N$. Hence, (3.46) yields, using Stirling's formula again,

$$
\begin{aligned}
\frac{Z_{N}}{(N-1) !^{\alpha}} & =(1+o(1)) \sum_{\left(m_{i}\right) \in V} \exp \left\{\sum_{j=2}^{K} \frac{\alpha B^{j}-A^{j}}{j(j-1) N^{j-1}}\right\} \prod_{i=1}^{K} \frac{N^{m_{i}-i \alpha m_{i}} i !^{\alpha m_{i}}}{m_{i} !} \\
& =\sum_{\left(m_{i}\right) \in V} \exp \left(f\left(m_{1}, \ldots, m_{K}\right)+o(1)\right),
\end{aligned}
$$


where

$$
\begin{array}{r}
f\left(m_{1}, \ldots, m_{K}\right)=\sum_{i=1}^{K}( \\
\quad(1-\alpha i) m_{i} \log N+\alpha m_{i} \log (i !)-m_{i} \log m_{i} \\
\left.\quad+m_{i}-\frac{1}{2} \log \left(2 \pi m_{i}\right)\right)+\sum_{j=2}^{K} \frac{\alpha B^{j}-A^{j}}{j(j-1) N^{j-1}} .
\end{array}
$$

Regard $f$ as a function of real variables $m_{1}, \ldots, m_{K}$. Then, for $m_{1}, \ldots, m_{K} \in$ $V$, which entails $A, B=O\left(N^{1-\alpha}\right)$,

$$
\begin{aligned}
\frac{\partial f}{\partial m_{i}} & =(1-\alpha i) \log N+\alpha \log (i !)-\log m_{i}-\frac{1}{2 m_{i}}+\sum_{j=2}^{K} \frac{\alpha i B^{j-1}-A^{j-1}}{(j-1) N^{j-1}} \\
& =\log n_{i}-\log m_{i}-\frac{1}{2 m_{i}}+\frac{\alpha i B-A}{N}+O\left(N^{-2 \alpha}\right) \\
& =\log n_{i}-\log m_{i}-\frac{1}{2 m_{i}}-\frac{(1-i \alpha) m_{1}}{N}+O\left(N^{-2 \alpha}\right) \\
& =\log n_{i}-\log m_{i}+o(1)
\end{aligned}
$$

and, similarly,

$$
\frac{\partial^{2} f}{\partial m_{i} \partial m_{j}}=-\frac{\delta_{i j}}{m_{i}}+O\left(\frac{\delta_{i j}}{m_{i} m_{j}}\right)+O\left(\frac{1}{N}\right)
$$

$V$ is compact and $f$ continuous, so $f$ attains its maximum in $V$ at some point $\mathbf{n}^{*}=\left(n_{1}^{*}, \ldots, n_{K}^{*}\right) \in V$. By (3.57), for large $N, \frac{\partial f}{\partial m_{i}}>0$ when $m_{i}=(1-\eta) n_{i}$ and $\frac{\partial f}{\partial m_{i}}<0$ when $m_{i}=(1+\eta) n_{i}$, so the maximum is not attained on the boundary of $V$, i.e. $\left|n_{i}^{*}-n_{i}\right|<\eta n_{i}$. Consequently, by (3.57),

$$
0=\frac{\partial f}{\partial m_{i}}\left(\mathbf{n}^{*}\right)=\log n_{i}-\log n_{i}^{*}+o(1)
$$

and thus $n_{i}^{*}=(1+o(1)) n_{i}$. A Taylor expansion of $f$ at $\mathbf{n}^{*}$ yields, using (3.59) and (3.58), for $\mathbf{m}=\left(m_{1}, \ldots, m_{K}\right) \in V$,

$$
\begin{array}{r}
f(\mathbf{m})=f\left(\mathbf{n}^{*}\right)-\frac{1}{2} \sum_{i=1}^{K}\left(\frac{\left(m_{i}-n_{i}^{*}\right)^{2}}{n_{i}^{*}}+O\left(\frac{\left|m_{i}-n_{i}^{*}\right|^{2}+\left|m_{i}-n_{i}^{*}\right|^{3}}{n_{i}^{2}}\right)\right) \\
+O\left(\frac{\left|\mathbf{m}-\mathbf{n}^{*}\right|^{2}}{N}\right)
\end{array}
$$

Choosing $\eta$ small enough, this implies first (for large $N$ )

$$
f(\mathbf{m}) \leq f\left(\mathbf{n}^{*}\right)-\frac{1}{3} \sum_{i=1}^{K} \frac{\left(m_{i}-n_{i}^{*}\right)^{2}}{n_{i}^{*}},
$$


which implies that it suffices to consider terms in (3.56) with, say, $\left|m_{i}-n_{i}^{*}\right|<$ $n_{i}^{1 / 2} \log N$; let $V_{1} \subset V$ be the set of such $\mathbf{m}$. For such terms, (3.60) yields

$$
f(\mathbf{m})=f\left(\mathbf{n}^{*}\right)-\frac{1}{2} \sum_{i=1}^{K} \frac{\left(m_{i}-n_{i}^{*}\right)^{2}}{n_{i}^{*}}+o(1),
$$

and thus by (3.56), letting $\beta=f\left(\mathbf{n}^{*}\right)$ be the maximum value of $f$ on $V$,

$$
\frac{Z_{N}}{(N-1) !^{\alpha}}=(1+o(1)) \sum_{\left(m_{i}\right) \in V_{1}} \exp \left(\beta-\frac{1}{2} \sum_{i=1}^{K} \frac{\left(m_{i}-n_{i}^{*}\right)^{2}}{n_{i}^{*}}+o(1)\right) \text {. }
$$

Since each term here corresponds to the case $X_{i+1, N}=m_{i}, i=1, \ldots, K$, and $n_{i}^{*}=(1+o(1)) n_{i}$, (2.12) follows. Furthermore, (3.63) also yields the Poisson approximation result in Remark 2.7, since the Poisson probabilities $\mathbb{P}\left(Y_{i, N}=m_{i}, \forall i\right)$ can easily be approximated by the same Gaussian as in (3.63); we omit the details.

In order to obtain more precise estimates of $n_{i}^{*}$, we go back to (3.57) and refine (3.59) to

$$
0=\frac{\partial f}{\partial m_{i}}\left(\mathbf{n}^{*}\right)=\log n_{i}-\log n_{i}^{*}-\frac{(1-i \alpha) n_{1}^{*}}{N}+O\left(N^{-2 \alpha}+N^{i \alpha-1}\right)
$$

which yields

$$
\log \frac{n_{i}^{*}}{n_{i}}=-\frac{(1-i \alpha) n_{1}^{*}}{N}+O\left(N^{-2 \alpha}+N^{i \alpha-1}\right)
$$

and thus, recalling $n_{1}^{*} / N=O\left(N^{-\alpha}\right)$,

$$
\frac{n_{i}^{*}}{n_{i}}=1-\frac{(1-i \alpha) n_{1}^{*}}{N}+O\left(N^{-2 \alpha}+N^{i \alpha-1}\right)
$$

Taking $i=1$ we find $n_{1}^{*} / n_{1}=1+O\left(N^{-\alpha}\right)$, and thus $n_{1}^{*}-n_{1}=O\left(N^{1-2 \alpha}\right)$, so (3.66) yields

$$
\frac{n_{i}^{*}}{n_{i}}=1-\frac{(1-i \alpha) n_{1}}{N}+O\left(N^{-2 \alpha}+N^{i \alpha-1}\right)
$$

establishing (2.14).

We obtain (2.15) $-(2.20)$ from (2.12) and (2.14) by checking that in each case, the omitted terms in the numerator are of smaller order than the denominator.

Finally, to evaluate the partition function, we approximate the sum in (3.63) by a Gaussian integral and obtain

$$
\frac{Z_{N}}{(N-1) !^{\alpha}}=(1+o(1)) e^{\beta} \prod_{i=1}^{K} \sqrt{2 \pi n_{i}^{*}}=e^{\beta+o(1)} \prod_{i=1}^{K} \sqrt{2 \pi n_{i}} .
$$

We have $\beta=f\left(\mathbf{n}^{*}\right)$. Further, (3.67) shows $n_{i}^{*}-n_{i}=O\left(n_{i} N^{-\alpha}\right)=O\left(N^{1-2 \alpha}\right)$, and it follows from (3.60) that, with $\mathbf{n}=\left(n_{1}, \ldots, n_{K}\right)$,

$$
f(\mathbf{n})=f\left(\mathbf{n}^{*}\right)+O\left(N^{1-3 \alpha}\right)=\beta+O\left(N^{1-3 \alpha}\right),
$$


so it remains to evaluate $f(\mathbf{n})$. For $\mathbf{m}=\mathbf{n}$, the final sum in (3.56) is

$$
\frac{\alpha B^{2}-A^{2}}{2 N}+O\left(N^{1-3 \alpha}\right)=\frac{(\alpha-1) n_{1}^{2}}{2 N}+O\left(N^{1-3 \alpha}\right)
$$

and thus, after some cancellations,

$$
f(\mathbf{n})=\sum_{i=1}^{K}\left(n_{i}-\frac{1}{2} \log \left(2 \pi n_{i}\right)\right)-\frac{(1-\alpha) n_{1}^{2}}{2 N}+O\left(N^{1-3 \alpha}\right) .
$$

Hence, (3.68) yields, with (3.69) and (3.71) and recalling $n_{1}=N^{1-\alpha}$,

$$
\frac{Z_{N}}{(N-1) !^{\alpha}}=\exp \left(\sum_{i=1}^{K} n_{i}-\frac{1-\alpha}{2} N^{1-2 \alpha}+O\left(N^{1-3 \alpha}\right)+o(1)\right)
$$

We substitute $n_{1}$ and $n_{2}$ from (2.9) and drop $n_{i}$ for $i \geq 3$, which yields (2.22).

Acknowledgement. This research was done while the authors visited NORDITA, Stockholm, during the program Random Geometry and Applications, 2010 .

\section{REFERENCES}

[1] J. Ambjorn, B. Durhuus and T. Jonsson, Quantum Geometry: a Statistical Field Theory Approach. Cambridge University Press, Cambridge, 1997.

[2] B. Durhuus, Probabilistic aspects of infinite trees and surfaces. Acta Physica Polonica B 34 (2003), 4795-4811

[3] B. Durhuus, T. Jonsson and J. F. Wheater, The spectral dimension of generic trees. J. Stat. Phys. 128 (2007), 1237-1260.

[4] M. Dwass, The total progeny in a branching process and a related random walk. $J$. Appl. Probab. 6 (1969), 682-686.

[5] P. Flajolet and R. Sedgewick, Analytic Combinatorics. Cambridge Univ. Press, Cambridge, UK, 2009.

[6] T. Jonsson and S. Ö. Stefánsson, Condensation in nongeneric trees. Journal of Statistical Physics, 142 (2011), no. 2, 277-313.

[7] V. F. Kolchin, Random Mappings. Optimization Software, New York, 1986.

[8] R. Lyons, R. Pemantle and Y. Peres, Conceptual proofs of $L \log L$ criteria for mean behavior of branching processes. Annals of Probability 23 (1995), no. 3, 1125-1138.

[9] A. Meir and J. W. Moon, On the altitude of nodes in random trees. Canad. J. Math., 30 (1978), 997-1015.

[10] J. Pitman, Enumerations of trees and forests related to branching processes and random walks. Microsurveys in Discrete Probability (Princeton, NJ, 1997), DIMACS Series in Discrete Mathematics and Theoretical Computer Science, 41, Amer. Math. Soc., Providence, RI, 1998, pp. 163-180. 
Department of Mathematics, Uppsala University, PO Box 480, SE-751 06 UPPSAla, SWEDEN

E-mail address: svante.janson@math.uu.se

$U R L:$ http://www2.math.uu.se/ svante/

The Science Institute, University of Iceland, Dunhaga 3, 107 Reykjavik, ICELAND

E-mail address: thjons@raunvis.hi.is

NORDita, Roslagstullsbacken 23, SE-106 91 Stockholm, Sweden

E-mail address: sigste@nordita.org 